\title{
ACTIVIDADES FORMATIVAS DE DISEÑO GRÁFICO ORIENTADAS A LA ADQUISICIÓN DE COMPETENCIAS PROFESIONALES: LAS TERCERAS JORNADAS DE PERIODISMO AUDIOVISUAL
}

\author{
Carlos Jiménez-Narros ${ }^{1}$ : Universidad Nebrija. España \\ cjimenez@nebrija.es
}

\section{RESUMEN}

La Universidad Nebrija y en concreto la facultad de Comunicación siempre ha apostado por la realización de experiencias formativas muy relacionadas con el mundo profesional, sirvan de ejemplo actividades como el Festival Jóvenes Tocados por la Publicidad, El Festival de Cortos ADN y El Concurso Periodístico Nipho. han desarrollado durante el curso académico 2010/2011 concretamente en las asignaturas de Elementos del diseño gráfico (grado en Periodismo) y Herramientas del diseño publicitario (grado en Publicidad). Las jornadas fueron aprovechadas para la formación específica de los alumnos en los dos perfiles profesionales mencionados. Los estudiantes de periodismo tenían que confeccionar una doble página cuyos contenidos eran una entrevista y una crónica; y los de publicidad debían diseñar un cartel publicitario. El objetivo último de ambas experiencias formativas era convertir el aula en un espacio vivo de aprendizaje. En esta ocasión el propósito de este artículo es integrar las terceras Jornadas de Periodismo Audiovisual como una actividad académica dirigida desde el aula de diseño gráfico. Los casos reales a los que haré referencia y que deseo compartir se

PALABRAS CLAVE: Diseño Gráfico - actividades académicas - experiencias formativas - competencias profesionales - periodismo audiovisual

\footnotetext{
${ }^{1}$ Autor correspondiente

Carlos Jiménez-Narros: Profesor Doctor del Grado en Periodismo. Universidad Nebrija. Madrid, España.

Correo: cjimenez@nebrija.es
} 


\title{
GRAPHIC DESIGN TRAINING ACTIVITIES AIMED AT THE ACQUISITION OF PROFESSIONAL SKILLS: THE THIRD CONFERENCE OF AUDIOVISUAL JOURNALISM
}

\begin{abstract}
Nebrija University and specifically the School of Communication has always bets on making formative experiences closely related to the professional world, for example, festivals as Young people touched by advertising, Festival of Short Films AdN and the Journalism Competition Nipho. This time the purpose of this article is to integrate the third conference of Audiovisual Journalism as an academic activity directed from the graphic design classroom. Real cases to which I refer and I wish to share have been developed during the academic year 2010/2011, specifically, in the subjects of Graphic design elements (degree in Journalism) and Advertising Design Tools (degree in Advertising). The conference served for specific training of students in the two professional profiles previously mentioned. The journalism students had to make a double page whose content was an interview and a chronic; advertising students had to design an advertising poster. The ultimate goal of both formative experiences was to turned the classroom in a living espace of learning.
\end{abstract}

KEY WORDS: Graphic Design - academic activities - learning experiences professional skills - audiovisual journalism

\section{INTRODUCCIÓN}

\subsection{Las III Jornadas de Periodismo Audiovisual}

Durante el curso académico 2010-2011 el Campus de La Berzosa de la Universidad Antonio de Nebrija ha sido el escenario de las III Jornadas de Periodismo Audiovisual, en esta edición centradas en "La fuerza del deporte". El título elegido ha sido en homenaje al éxito del deporte español. La tercera edición se dividió en dos actos: el 24 de noviembre de 2010 y el 5 de abril de 2011.

La primera parte de estas jornadas -24 de noviembre de 2010- reunió a figuras de primer nivel del periodismo deportivo en televisión, como Antonio Lobato, Julio Morales y Óscar del Castillo, integrantes del equipo de comentaristas de la Fórmula 1 de La Sexta televisión; Nico Abad, presentador de los deportes de Cuatro televisión; y profesionales de la sección de deportes de Televisión Española, como el comentarista Perico Delgado, campeón del Tour de Francia de 1988, el presentador Marcos López e Ignacio Gómez-Acebo, director de deportes.

La segunda jornada -5 de abril de 2011- consistió en que los asistentes (profesores, estudiantes y profesionales del deporte) conocieran por dentro cómo funciona el programa Marcagol de Marca TV (La Sexta). Presenciaron el simulacro de su programa en diresto. narrado desde la óntica más divertida v en $11 n$ tono distendido. oenerado 
por la magnífica sintonía que existe entre todos los miembros del equipo; los presentadores Juan Antonio Villanueva y Lara Álvarez, el editor Rosendo López, el realizador Roberto Gómez y el exfutbolista y comentarista deportivo Daniel García.

Como novedad y para dar la sensación de más realismo, las III Jornadas de Periodismo Audiovisual se celebraron en el plató de televisión de la facultad de Ciencias de la Comunicación del Campus de La Berzosa. Estudiantes, profesores y demás asistentes pudieron disfrutar de las experiencias de profesionales que cubren día a día la información deportiva en televisión, en un momento en que el deporte nacional ocupa un buen lugar en el palmarés internacional, con gran protagonismo en los medios escritos y muchas horas en la programación de los audiovisuales.

Aparte de la vertiente profesional consustancial a este tipo de encuentros, las jornadas estaban diseñadas con el objetivo de convertirse en una experiencia interdisciplinar para los estudiantes del grado en Periodismo y Publicidad en el nuevo marco del Espacio Europeo de Educación Superior (EEES)

\section{METODOLOGÍA}

\subsection{Periodistas-diseñadores}

La primera práctica realizada con los alumnos de periodismo tenía como propósito interrelacionar las asignaturas de Elementos del diseño gráfico y Redacción periodística informativa (ambas materias se imparten en segundo curso), con el fin de que los estudiantes se convirtieran durante esos dos días y luego, a lo largo de varias sesiones de estas dos asignaturas, en verdaderos profesionales de los medios. Durante las jornadas eran reporteros que cubrían en directo lo que en ellas pasaba. En las clases de redacción convertían los dichos y hechos en crónicas y entrevistas a uno de los "personajes relevantes" del deporte, para acabar dándoles forma y figura en el diseño de una doble página (enfrentada) en la asignatura de Elementos del diseño gráfico.

Como docente estoy convencido de que no hay mejor experiencia que hacer sentir la profesión lo antes posible a los estudiantes, para que cuando realmente trabajen en un medio de comunicación, bien como periodistas, bien como diseñadores, comprueben que la vida profesional entró de lleno en las aulas durante sus primeros años de formación.El departamento de Periodismo bajo esta misma filosofía de trabajo organiza cada año el Concurso Periodístico Nipho ${ }^{2}$

Una vez que los alumnos habían acabado sus trabajos redaccionales de crónicas y entrevistas, -acompañados del material fotográfico- tenían que diseñar para una revista, una doble página enfrentada (formato DIN-A4) con un aspecto visual

\footnotetext{
2 Jiménez Narros, C. \& Cachán Alcolea, C. (2010). El concurso periodístico Nipho: una experiencia formativa en el Espacio Europeo de Educación Superior (EEES). En Sánchez, J. \& González, J. (coords.) Métodos de Innovación docente aplicados a los estudios de Ciencias de la Comunicación. Madrid: Fragua, pp. 170_101
} 
atractivo. Indicar que hasta ese momento los estudiantes sólo habían confeccionado páginas sueltas -espacio vertical-; de esta manera pasaron a trabajar sobre un espacio horizontal

Hecha esta aclaración obligada, señalamos que los objetivos de diseño perseguidos con dicha práctica real son:

a) Elegir una retícula acorde al formato, considerando: márgenes, número de columnas, anchura de los medianiles, cabeceras de sección y foliación. Se categoriza así, la información, se distribuyen los espacios en blanco, se racionaliza el espacio y se concede orden y continuidad a los temas de actualidad.

b) Trabajar con texto redactado y real, olvidando el texto de relleno o ficticio. Esta novedad supone que el alumno-maquetador tiene que ajustarse al espacio del que disponible y para ello debe poner en marcha toda la creatividad posible, ampliar o disminuir los diferentes elementos: antetítulos, titulares, subtítulos, fotografías, cabeceras de sección y pies de foto, entre otros. En algunas ocasiones los autores de las fotografías eran los propios alumnos-redactores, lo que implica poner a prueba otras competencias adquiridas, en su primer curso, en la asignatura de Periodismo gráfico. El reto era editar los textos de manera correcta en tamaño, estilo, formato, sangrías y alineaciones.

c) Diferenciar visualmente dos géneros periodísticos: crónica y entrevista. Este planteamiento supone conocer con qué materiales contamos y así distribuir los espacios e impactos visuales. Confeccionar la entrevista conlleva unos condicionantes estéticos específicos; preguntas del futuro periodista, respuestas y fotografía del entrevistado. En el caso de la crónica no se definieron condicionantes.

d) Utilizar la tipografía y el color como elementos de diseño. Hay que buscar armonía y contraste tipográfico entre antetítulos, titulares, subtítulos, textos, entradillas, pies de foto y capitulares. En las dos páginas proyectadas, el color hay que plantearlo como guía de navegación, facilitando el recorrido visual, reforzando la lectura, creando jerarquías y armonía visual.

La intención última de esta actividad es que los alumnos manejen con soltura Indesig, el programa estrella en diseño editorial. El profesor durante las sesiones docentes se revela como guía -académico y profesional- que corrige y potencia las propuestas planteadas, y en ocasiones muestra nuevas fórmulas o modelos.

\subsection{Concurso de carteles publicitarios}

La segunda experiencia formativa -referida a las mismas jornadas- se desarrolla con los alumnos del grado en Publicidad.

El departamento de Periodismo y Comunicación Audiovisual que organizaba el encuentro entregó un briefing real, con los objetivos de comunicación. 
El encargo consistía en construir un cartel a tamaño DIN A-3 el que se informaba de la visita de los responsables del programa Marcagol, de Marca TV (La Sexta). De esta manera se convocaba a todos los interesados y se les animaba a asistir.

Al tratarse de un cartel informativo existían una serie de elementos gráficos obligatorios (nombre de las jornadas, ponentes, entidades colaboradoras, día, lugar y hora de la convocatoria). Para la realización de las piezas, podían elegir el estilo de cartel deseado. En la confección de los bocetos y artes finales, los estudiantes manejaron principalmente dos aplicaciones de diseño gráfico, Photoshop e Illustrator.

Los alumnos de Publicidad que cursan la asignatura Herramientas del diseño publicitario trabajaron en equipo y plantearon sus apuestas gráficas al departamento organizador, que finalmente valoró cuál era el cartel más original y apropiado para publicar. Las soluciones gráficas a las que han llegado los alumnos han sido muy interesantes, creativamente hablando.

Mientras unos apuestan por el cómic (Daniel Polo y Álvaro Odriozola), otros prefieren carteles puramente tipográficos (Sara González e Isabel Albelda), o más visuales (Raúl del Cuadro, Eva Zamorano, Mari Cielo Medina); sin olvidar al equipo ganador formado por Noelia Dorado y Verónica Conde que plantearon el cartel de las jornadas deportivas como una partida de chapas.

Una vez elegida la idea creativa ganadora la pieza fue adaptada a la línea gráfica de la Universidad.
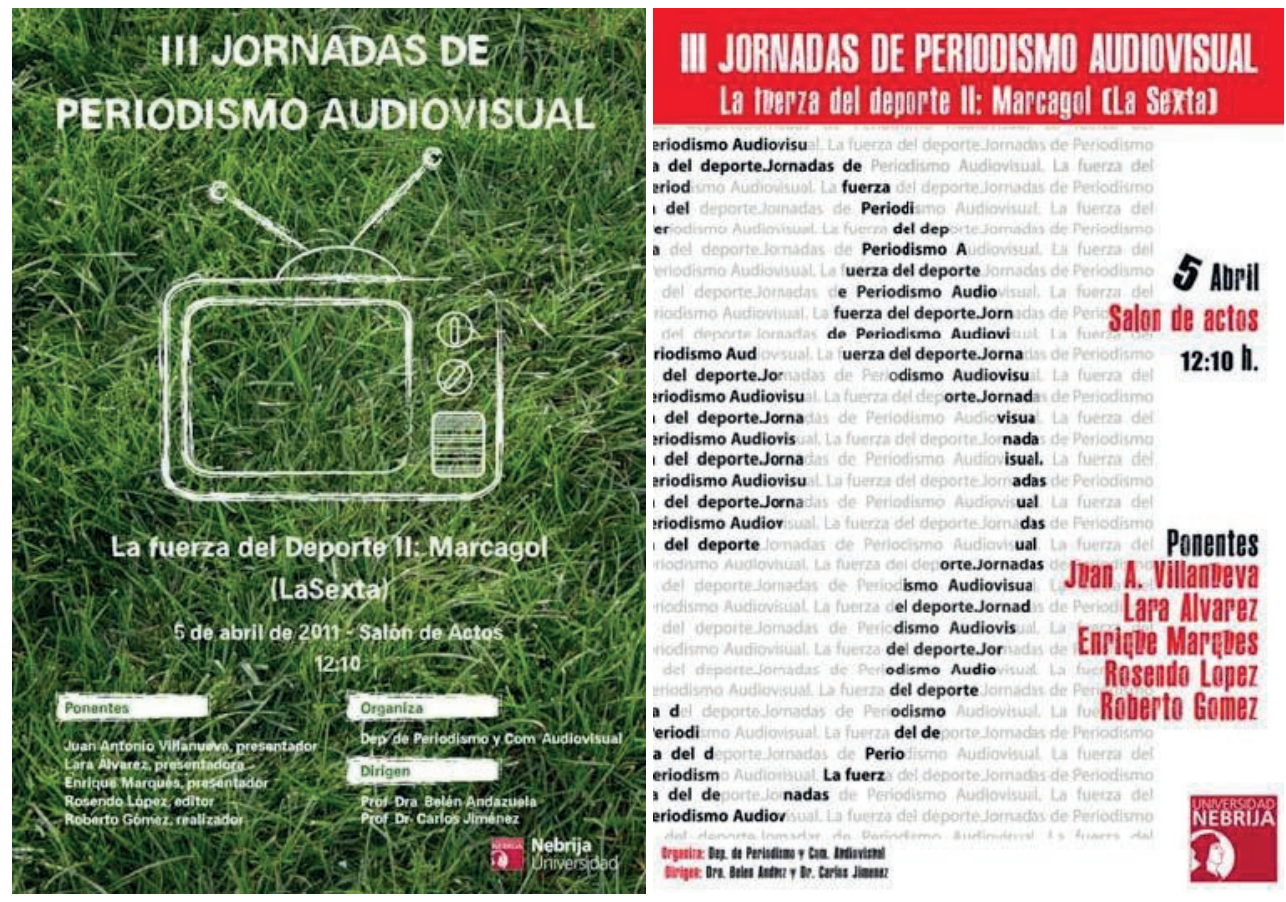

Figura 1. Carteles diseñados por alumnos de publicidad: Raúl del Cuadro (izquierda), Sara González e Isabel Albelda (derecha) 


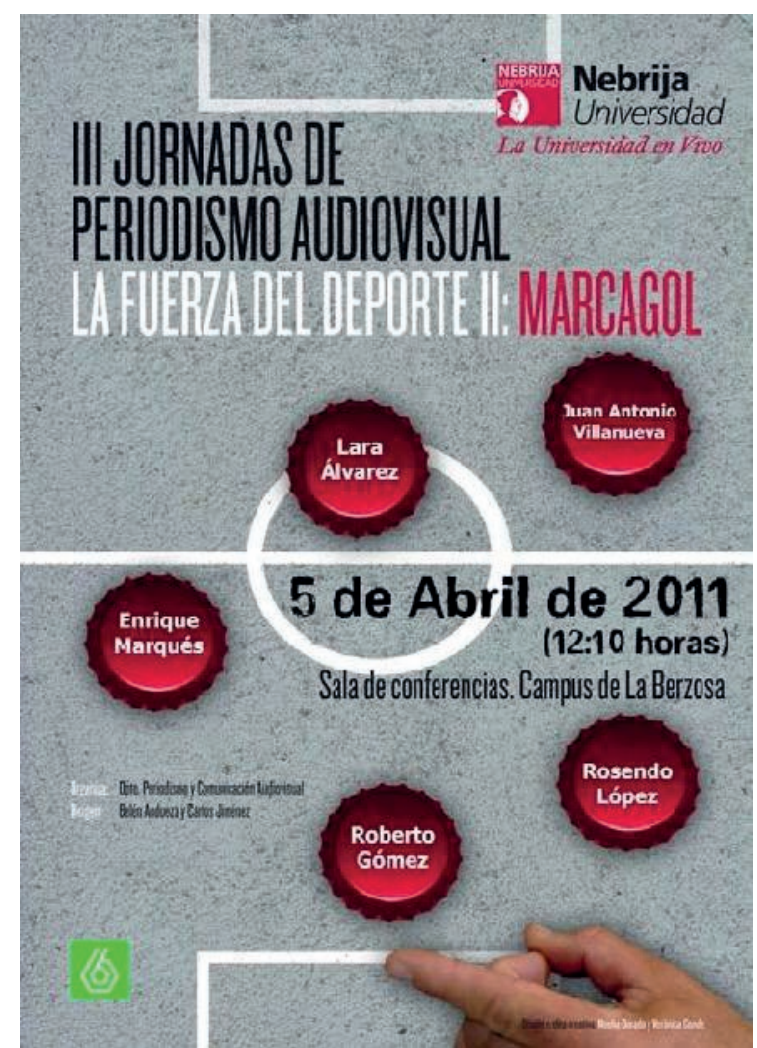

Figura 2. Cartel ganador diseñado por Noelia Dorado y Verónica Conde, alumnas de segundo de publicidad

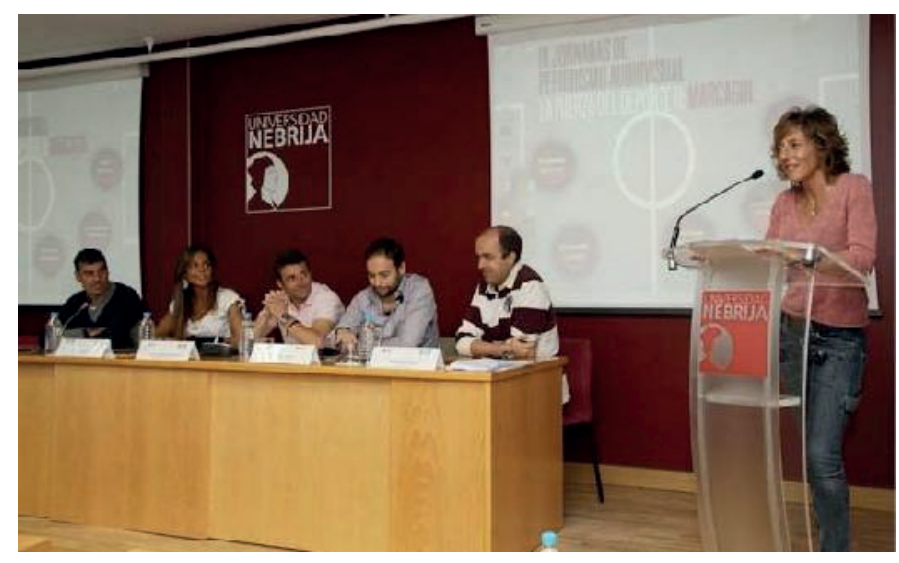

Figura 3. La profesora Belén Andueza presenta al equipo del programa Marcagol durante su visita a la Nebrija. Sentados en la mesa (de izqda a dcha): Daniel García, Lara Álvarez, Juan Antonio Villanueva, Roberto Gómez y Rosendo López (Foto: Zaida Del Río) 


\section{CONCLUSIONES}

El nuevo Espacio Europeo de Educación Superior (EEES) alienta la puesta en marcha de actividades dirigidas, en las que los estudiantes no sólo adquieren competencias transversales, sino también profesionales, familiarizándose con su futura actividad laboral. En este sentido la Universidad Nebrija y en concreto la facultad de Comunicación, organiza desde hace años, varias actividades para acercar la realidad profesional a los estudiantes. Cada titulación organiza una actividad extraacadémica relevante, Publicidad (Festival Jóvenes Tocados por la Publicidad), Periodismo (Concurso Periodístico Nipho) y Comunicación Audiovisual (Festival de Cortos ADN).

Este modelo de experiencias formativas son la prueba más fehaciente de que las prácticas dentro del aula deben ser lo más reales y cercanas posibles, por la mayor implicación de los estudiantes en el proceso de aprendizaje. Muchas gracias a todos los alumnos de Periodismo y Publicidad del curso académico 2010-2011, que desde el principio recibieron con gran interés y entusiasmo las actividades planteadas.

\section{REFERENCIAS}

Herrán, A. (2005). Formación y transversalidad universitarias. Tendencias Pedagógicas (10), pp.223-256. Recuperado el 05 de Diciembre de 2011, de www.tendenciaspedagogicas.com/Articulos/2005_10_11.pdf

Perlado Lamo De Espinosa, M. \& Rubio Romero, J. (2010). Experiencias docentes sobre actividades académicas orientadas a la adquisición de competencias transversales y profesionales de los estudiantes de grado en Publicidad. En Sánchez, J. \& Lorenzo, F. (coords.) Competencias y perfiles profesionales en los estudios de Ciencias de la Comunicación. Madrid: Fragua, pp173-183

\section{Carlos Jiménez-Narros}

Doctor por la Universidad Complutense de Madrid. Licenciado en Periodismo por la Facultad de Ciencias de la Información. Coordinador los estudios de grado en Periodismo de la Universidad Nebrija. Coordinador de Calidad de la facultad de Ciencias de la Comunicación (2008-junio 2011). Responsable del Concurso de Gráfica Nebrija y de su edición digital hasta el año 2009. Coordinador del departamento de creatividad del Festival Jóvenes Tocados por la Publicidad (2009). Creador del diseño de las revistas: Aida y Nuestra. Ponencias y comunicaciones publicadas en distintos congresos internacionales sobre comunicación, diseño y docencia. El área de investigación son las nuevas tecnologías de la información. Especialidad: diseño editorial y publicitario 